\title{
Synchronization and chaos in systems of coupled inner-ear hair cells
}

\author{
Justin Faber $\odot,{ }^{1, *}$ Hancheng Li $\odot,{ }^{2, \dagger}$ and Dolores Bozovic ${ }^{1,3, \ddagger}$ \\ ${ }^{1}$ Department of Physics \& Astronomy, University of California, Los Angeles, California 90095, USA \\ ${ }^{2}$ Department of Electrical \& Computer Engineering, University of California, Los Angeles, California 90095, USA \\ ${ }^{3}$ California NanoSystems Institute, University of California, Los Angeles, California 90095, USA
}

(Received 11 December 2020; accepted 15 February 2021; published 22 March 2021)

\begin{abstract}
Hair cells of the auditory and vestibular systems display astonishing sensitivity, frequency selectivity, and temporal resolution to external signals. These specialized cells utilize an internal active amplifier to achieve highly sensitive mechanical detection. One of the manifestations of this active process is the occurrence of spontaneous limit-cycle motion of the hair-cell bundle. As hair bundles under in vivo conditions are typically coupled to each other by overlying structures, we explore the role of this coupling on the dynamics of the system, using a combination of theoretical and experimental approaches. Our numerical model suggests that the presence of chaotic dynamics in the response of individual bundles enhances their ability to synchronize when coupled, resulting in significant improvement in the system's ability to detect weak signals. This synchronization persists even for a large frequency dispersion and when tens of oscillators comprise the system. Further, the amplitude and coherence of the active motion are not reduced upon increasing the number of oscillators. Using artificial membranes, we impose mechanical coupling on groups of live and functional hair bundles, selected from in vitro preparations of the sensory epithelium, allowing us to explore the role of coupling experimentally. Consistent with the numerical simulations of the chaotic system, synchronization occurs even for large frequency dispersion and a large number of hair cells. Further, the amplitude and coherence of the spontaneous oscillations are independent of the number of hair cells in the network. We therefore propose that hair cells utilize their chaotic dynamics to stabilize the synchronized state and avoid the amplitude death regime, resulting in collective coherent motion that could play a role in generating spontaneous otoacoustic emissions and an enhanced ability to detect weak signals.
\end{abstract}

DOI: 10.1103/PhysRevResearch.3.013266

\section{INTRODUCTION}

The auditory and vestibular systems exhibit remarkable sensitivity, frequency selectivity, and temporal resolution [1]. These systems can detect vibrations that induce motion of only a few angstroms, well below the amplitude induced by thermal fluctuations in the surrounding fluid. Humans are able to distinguish sounds that differ in frequency by only $\sim 0.2 \%$. Further, we are able to resolve two stimulus impulses that differ temporally by only 10 microseconds [2]. These characteristics are crucial for identifying and localizing sounds, as well as comprehending speech, especially in noisy environments. These phenomena, among others, are not fully understood and the physics of hearing remains an active area of research [3].

Mechanical detection of auditory signals is performed by hair cells. These specialized sensory cells are named after

\footnotetext{
*faber@physics.ucla.edu

†hanchengli@ucla.edu

*bozovic@physics.ucla.edu
}

Published by the American Physical Society under the terms of the Creative Commons Attribution 4.0 International license. Further distribution of this work must maintain attribution to the author(s) and the published article's title, journal citation, and DOI. the rodlike stereovilli that protrude from their apical surface. The stereovilli are arranged in interconnecting rows and are collectively named the hair bundle. Incoming sound waves or vestibular accelerations induce deflections of the hair bundle, which cause a shearing motion between neighboring stereovilli. This shearing causes mechanically gated ion channels to open, yielding an influx of ionic current into the hair cell [4-6]. The resulting changes in the membrane potential elicit further signaling from the hair cell to the auditory neurons, propagating the information that a mechanical signal has been detected.

Auditory detection has been shown to require an active, energy-consuming process [7]. In some nonmammalian species, hair bundles have been further shown to oscillate spontaneously in the absence of external stimulus [8-10]. These limit-cycle oscillations exhibit amplitudes significantly larger than the motion induced by the thermal fluctuations of the surrounding fluid, and they have been shown to violate the fluctuation-dissipation theorem, proving them to be active [11]. The existence and role of these spontaneous oscillations in intact animals has not yet been established. However, the results of several studies suggest that they could be important for signal detection, as they provide a potential amplification mechanism [12]. Further, spontaneously oscillating hair bundles provide a probe for studying the underlying active processes of the inner ear. 
Given the existence of essential nonlinearities in the auditory system, nonlinear dynamics theory has been applied to study its behavior. Specifically, the dynamics of individual hair bundles have been well described with the normal form of the Hopf bifurcation [13,14]. This simple differential equation reproduces many of the experimentally observed features of the hair-cell dynamics, such as the sensitivity and frequency selectivity, as well as the spontaneous oscillations and the compressive nonlinear response. To achieve this extreme sensitivity and frequency selectivity, the system has been assumed to be poised at a Hopf bifurcation. However, in the proximity of this bifurcation, the system experiences a phenomenon known as critical slowing down, meaning that a stimulus perturbing it away from the steady-state behavior will result in a long transient before returning to the steady state [15]. This is inconsistent with the high temporal resolution of the auditory system. To avoid the inherent trade-off between sensitivity and temporal resolution, we proposed that the system is poised deeply in the oscillatory regime, rather than in the immediate vicinity of the Hopf bifurcation. We have previously shown that a system which exhibits chaotic dynamics in the oscillatory regime shows an enhancement of both sensitivity and rapidity of response [16,17]. However, in this dynamical regime, an individual uncoupled oscillator is not frequency selective.

In vivo, hair bundles are mechanically coupled by an overlying membrane. The nature of this coupling varies across species and across the organs of the inner ear [6]. It tends to be strong and, in some cases, may suppress the spontaneous oscillations. However, the inner ear does spontaneously emit faint tones in the absence of stimulus [18]. These spontaneous otoacoustic emissions (SOAEs) are ubiquitous across vertebrate species and occur only in live animals with intact inner ears, suggesting that they arise from an active process [19]. The mechanism responsible for their production has not yet been established, but several theoretical studies suggest they may arise from the spontaneous motion of actively oscillating coupled hair bundles, through a phenomenon known as frequency clustering [20,21]. For actively oscillating hair bundles to produce SOAEs, they would need to overcome a phenomenon known as amplitude death, which occurs when active oscillators with large frequency dispersion are strongly coupled, resulting in quenching of the motion [22]. Further, hair bundles with different characteristic frequencies would need to be able to synchronize in order to form the narrow spectral peaks found in SOAE recordings.

We have previously demonstrated a mechanism by which chaos can aid in an oscillator's ability to synchronize to external signals $[23,24]$. In the current work, we extend this study to a system of coupled active oscillators, which provides a model for the behavior of a full auditory or vestibular end organ. Specifically, we show that this same chaotic regime causes Hopf oscillators to avoid amplitude death and instead synchronize with each other, despite large dispersion in the characteristic frequencies. We show that this synchronization is stable, as it persists for large system sizes, providing a plausible model for biological systems. Neither the amplitude nor the coherence of the spontaneous motion is compromised upon increasing the number of oscillators in the network. We test these theoretical predictions by experimental studies performed on in vitro preparations of excised epithelia, in which hair bundles were coupled using artificial membranes. We find consistent results in our experimental studies and theoretical predictions. Therefore, we propose that chaotic dynamics enhance the synchronization of oscillating hair bundles, causing the system to avoid the amplitude death state and instead produce spontaneous motion that could aid in signal detection, as well as result in the production of SOAEs.

Using the numerical model of this coupled system, we also demonstrate that this chaos-induced synchronization results in enhanced sensitivity and frequency selectivity to weak, external signals without compromising the speed of the response. This mechanism provides an attractive alternative to the dynamical regime in the immediate vicinity of the Hopf bifurcation, where the system sacrifices temporal resolution due to critical slowing down.

\section{NUMERICAL MODEL OF COUPLED HAIR BUNDLE DYNAMICS}

The dynamics of the $j$ th oscillator in the system are governed by the normal form equation for the supercritical Hopf bifurcation,

$$
\begin{aligned}
\frac{d z_{j}(t)}{d t}= & \left(\mu+i \omega_{j}\right) z_{j}(t)-\left(\alpha+i \beta_{j}\right)\left|z_{j}(t)\right|^{2} z_{j}(t) \\
& +k\left[S(t)-x_{j}(t)\right]+F_{j}(t),
\end{aligned}
$$

where

$$
z_{j}(t)=x_{j}(t)+i y_{j}(t) .
$$

Here, $x_{j}(t)$ represents the bundle position, while $y_{j}(t)$ reflects internal parameters of the bundle and is not assigned a specific measurable quantity. However, the existence of this hidden variable is essential to reproduce the experimentally observed dynamics. $\mu$ represents the control parameter of the oscillators, which determines the proximity to the Hopf bifurcation. The natural frequency at this bifurcation point is given by $\omega_{j}$. For an individual, uncoupled oscillator, the limit-cycle radius is given by $r_{0}=\sqrt{\frac{\mu}{\alpha}}$, and the limit-cycle frequency at finite radius is $\Omega_{j}=\omega_{j}-\beta_{j} r_{0}^{2} . F_{j}(t)$ represents a real-valued external forcing on the $j$ th oscillator.

All oscillators are coupled to the overlying artificial membrane with coupling stiffness, $k$. The position of the membrane, $S(t)$, is governed by the differential equation,

$$
m \frac{d^{2} S(t)}{d t^{2}}+\lambda \frac{d S(t)}{d t}=\sum_{j=1}^{N} k\left[x_{j}(t)-S(t)\right],
$$

where $m$ and $\lambda$ represent the mass and drag of the artificial membrane, respectively.

Here, $\alpha$ and $\beta_{j}$ characterize the nonlinear term of the system. In most prior studies, $\beta_{j}$ was set to zero, rendering the oscillators isochronous. For such a system, the frequency is independent of the amplitude of the oscillation. However, when $\beta_{j} \neq 0$, the system is nonisochronous, and the instantaneous frequency depends on the amplitude of the oscillations. This results in more complex behavior and causes the additive noise to induce chaotic dynamics in the individual oscillators [16]. 
Hair bundle dynamics occur at a Reynolds number much below one [25]. This allows us to ignore the inertial forces of the artificial membrane $(m=0)$. Since the drag of the membrane is fairly small in comparison to the drag of the hair bundles (see the Appendix), we choose $\lambda=0.1$. We set $\mu=\alpha=1$, poising the system in the oscillatory regime. We use a significant coupling stiffness of $k=2$, which can lead to synchronization of the oscillators. We vary $\beta_{j}, \Omega_{j}$, and $\omega_{j}$ throughout this study and define the limit-cycle frequencies of the slowest and fastest oscillators in a system of $N$ oscillators to be $\Omega_{1}$ and $\Omega_{N}$, respectively. The other oscillators have limit-cycle frequencies uniformly spaced between $\Omega_{1}$ and $\Omega_{N}$. All numerical simulations were performed using the fourthorder Runge-Kutta method with time steps of $10^{-3}$, unless otherwise stated.

\section{METHODS}

\section{A. Biological preparation}

Experiments were performed in vitro on hair cells of the American bullfrog (Rana catesbeiana) sacculus, an organ responsible for detecting low-frequency airborne and groundborne vibrations. Sacculi were excised from the inner ear of the animal and mounted in a two-compartment chamber with artificial perilymph and endolymph solutions [8]. Hair bundles were accessed after digestion and removal of the overlying otolithic membrane [11]. All protocols for animal care and euthanasia were approved by the UCLA Chancellor's Animal Research Committee in accordance with federal and state regulations.

\section{B. Artificial membranes}

Mica powder was added to a vial of artificial endolymph solution. This solution was thoroughly mixed and then filtered through several steel mesh gratings. These gratings served as bandpass filters to separate the mica flakes into several desired sizes. This process was expedited by using vacuum suction to pull the solution through the grating. The solution containing the artificial membranes was pipetted into the artificial endolymph solution, above the biological preparation. Many of the membranes would land in the desired orientation and adhere to hair bundles underneath. These hair bundles could then be imaged through the transparent artificial membranes.

\section{Data collection}

Hair bundle motion was recorded with a high-speed camera at frame rates between 250 and 1000 frames per second. The records were analyzed in MATLAB using a center-of-pixelintensity technique to determine the position of the center of the hair bundle in each frame. The motion was tracked along the direction of increasing stereovilli height. Typical noise floors of this technique, combined with stochastic fluctuations of the bundle position in the fluid, were $3-5 \mathrm{~nm}$.

\section{Cross-correlation coefficient}

We characterize synchronization between spontaneously oscillating hair bundles using the cross-correlation coefficient,

$$
C\left[x_{1}(t), x_{2}(t)\right]=\frac{\left\langle\tilde{x}_{1}(t) \tilde{x}_{2}(t)\right\rangle}{\sigma_{1} \sigma_{2}},
$$

where $\tilde{x}_{1}(t)=x_{1}(t)-\left\langle x_{1}(t)\right\rangle$ and $\tilde{x}_{2}(t)=x_{2}(t)-\left\langle x_{2}(t)\right\rangle$ represent the time traces of the motion, $\sigma_{1}$ and $\sigma_{2}$ represent their respective standard deviations, and the angled brackets denote the time average. $C=1$ indicates perfectly correlated motion, while $C \approx 0$ is indicative of completely uncorrelated motion. We find the noise floor on this measure by calculating $C$ between 1225 unique pairs of uncoupled hair bundles. The histogram of these cross-correlation coefficients has a standard deviation of approximately 0.02 , with no points exceeding 0.1 (Fig. 6). To consider a pair of hair bundles to be coupled, we define our threshold to be $C \geqslant 0.1$, which is five standard deviations above the mean.

\section{E. Correlation time}

We measure the coherence of the spontaneous oscillations, which can be characterized by integrating the squared autocorrelation function to compute the correlation time [26],

$$
T_{c}(x(t))=\int_{0}^{\infty}\left[\frac{\left\langle\tilde{x}(t) \tilde{x}\left(t+t^{\prime}\right)\right\rangle}{\sigma^{2}}\right]^{2} d t^{\prime} .
$$

Due to the finite length of the experimental recordings, we truncate the integration at two mean periods of the spontaneous oscillations. We choose this duration, as the oscillations in the autocorrelation function have typically decayed after two full periods, and further integration would introduce unnecessary noise into the measure. Further, we scale this measure to the correlation time of a sine wave,

$$
\tau_{\mathrm{cor}}=\frac{T_{c}(x(t))}{T_{c}(\sin (t))} .
$$

Therefore, perfectly sinusoidal motion yields $\tau_{\text {cor }}=1$, while white Gaussian noise yields $\tau_{\text {cor }} \approx 0$.

\section{THEORETICAL RESULTS}

A nonisochronous system can modify its oscillation frequency by adjusting its amplitude, thus allowing it to easily entrain to off-resonant frequencies. As a result, two coupled oscillators with large frequency dispersion can synchronize. Further, if the degree of nonisochronicity of the oscillators differs in correspondence with the dispersion of characteristic frequencies, synchronization can be greatly enhanced in systems of many oscillators. In Figs. 1(c)-1(e), we illustrate this effect by plotting the instantaneous angular frequency of uncoupled oscillators,

$$
\frac{d \theta_{j}}{d t}=\omega_{j}-\beta_{j} r_{j}^{2},
$$

as a function of the radius of the oscillations, $r_{j}$. We plot these curves for four oscillators with frequency dispersion and show that the curves intersect when we include dispersion in $\beta_{j}$. Oscillators tend to meet at or near the intersection points, with synchronization enhanced even if the curves do not all intersect at the same point. We perform simulations of the numerical model and compare the isochronous case $\left(\beta_{j}=0\right)$ to the nonisochronous case, where $\beta_{j}$ varies linearly between 0 and $\beta_{\max }$, in accordance with $\omega_{j}$. We set $\Omega_{1}=1$ and $\Omega_{N}=2 \sqrt{5} \approx 4.47$, choosing the values to be similar to 

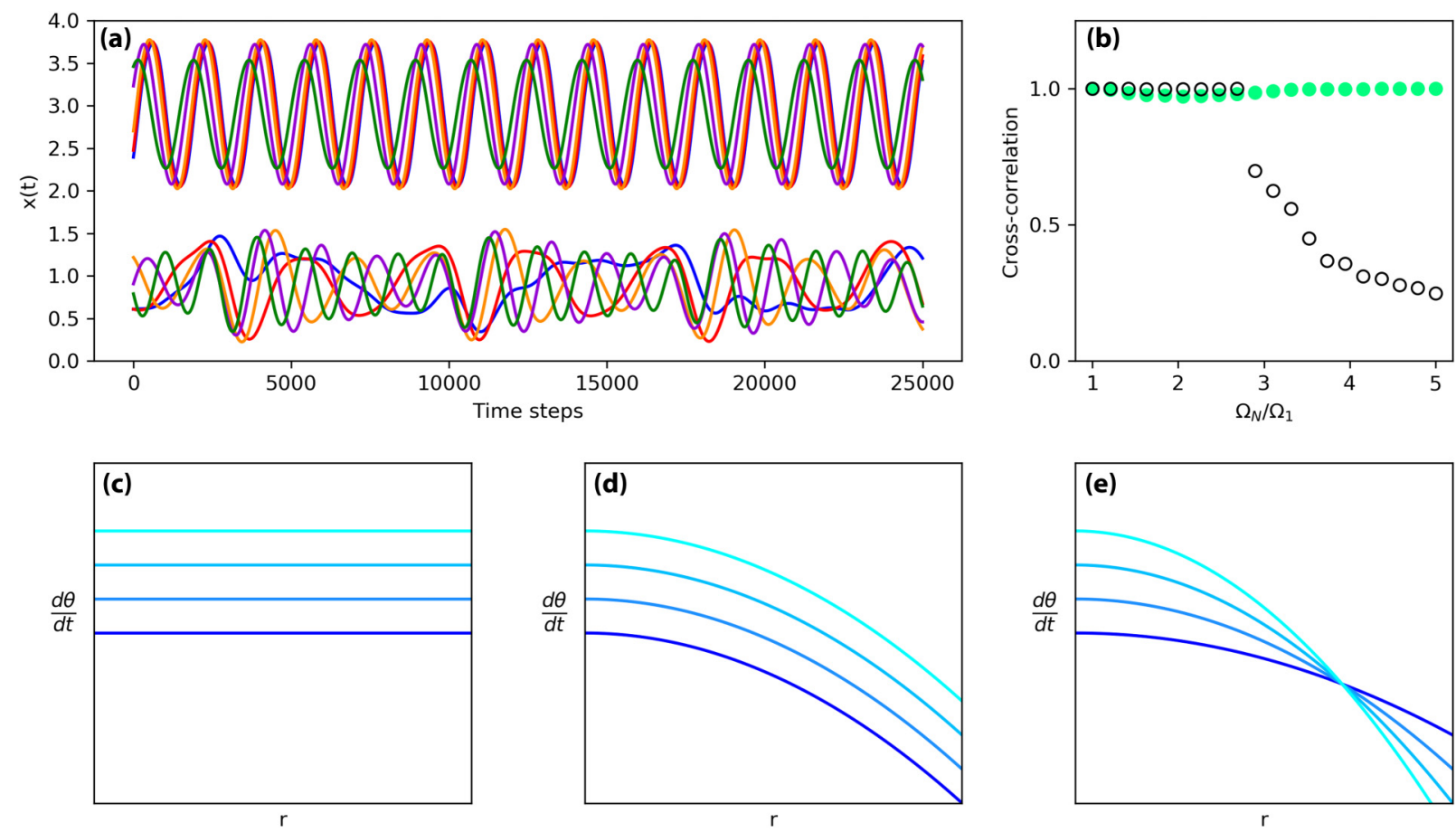

FIG. 1. (a) Time traces of five coupled isochronous oscillators (bottom) and five coupled nonisochronous oscillators with $\beta_{j}$ linearly spaced from 0 to 6 (top). (b) Average cross-correlation coefficient [Eq. (4)] between all pairs of oscillators as a function of the frequency dispersion. This system consists of five oscillators in the isochronous (black open circles) and nonisochronous (green filled circles) cases. (c)-(e) Illustrations of the instantaneous frequencies [Eq. (7)] of four oscillators as a function of the oscillation amplitude for the isochronous, nonisochronous with identical $\beta_{j}$, and nonisochronous with dispersion in $\beta_{j}$ systems, respectively.

the frequency dispersion observed in the experiments. Sample traces of these simulations are plotted in Fig. 1(a). We simultaneously modify $\beta_{j}$ and $\omega_{j}$ to adjust the level of nonisochronicity, while keeping the limit-cycle frequencies $\Omega_{j}$ fixed.

We assess the stability of the synchronized state of five coupled Hopf oscillators by measuring the average crosscorrelation coefficient [Eq. (4)] between all pairs of oscillators as a function of the frequency dispersion. In the isochronous case, synchronization becomes unstable for large frequency dispersions, pushing the system into the incoherent state. Upon an increase in the coupling strength, the isochronous system transitions into the amplitude death regime and the system becomes quiescent. However, in the nonisochronous system, the synchronized state persists even with fivefold frequency dispersion [Fig. 1(b)]. Further, for the nonisochronous system, the stability of the synchronized state preserves the amplitude (root mean square) and coherence [Eq. (6)] of the oscillators, rendering these measures independent of the system size (Fig. 2). This is in contrast to the isochronous system, for which the oscillation amplitude and coherence fall off with increasing network size.

We next determine the effects of nonisochronicity on the system's ability to detect weak signals. We apply a weak Gaussian white-noise stimulus, $F_{j}(t)=\eta_{j}(t)$, to each of the bundles. The noise terms of the oscillators are independent, with correlation function, $\left\langle\eta_{j}(t) \eta_{j}\left(t^{\prime}\right)\right\rangle=2 D \delta\left(t-t^{\prime}\right)$. We use a noise strength of $D=0.01$ and integrate the stochastic differential equations using the second-order Runge-Kutta method. We then calculate the power spectrum of the response of the oscillator, $x_{(N+1) / 2}$, which displays the median natural frequency. This method assumes the noise strength to be small enough to warrant consideration of only the linear response of the system.

The nonisochronous system exhibits much higher sensitivity and simultaneously provides a more narrow bandpass filter on the white-noise stimulus in comparison to the isochronous system [Figs. 3(a) and 3(b)]. We quantify the increase in sensitivity by finding the maximum value in the power spectrum and normalizing it by the maximum value of the power spectrum of the isochronous system. This measure of gain indicates the factor by which nonisochronicity enhances the sensitivity of the system [Fig. 3(d)]. Likewise, we calculate the quality factor of these peaks and normalize them by the quality factor of the isochronous system [Fig. 3(e)]. We find that these measures of sensitivity and frequency selectivity increase with system size, consistent with prior theoretical studies [27]. For a system of 20 oscillators, the synchronization induced by nonisochronicity leads to a sensitivity increase of over 300-fold and a frequency selectivity increase of over 100 -fold.

Lastly, we show that this large enhancement in the sensitivity and frequency selectivity of response does not come at the cost of reduced temporal resolution, in contrast with close proximity to a Hopf bifurcation. We provide an abrupt step-function stimulus to the system and average the responses of all of the oscillators. We then calculate the time it takes for the averaged response to settle to a constant value. As the plateau value fluctuates, we calculate the time required to settle within five standard deviations of the mean 

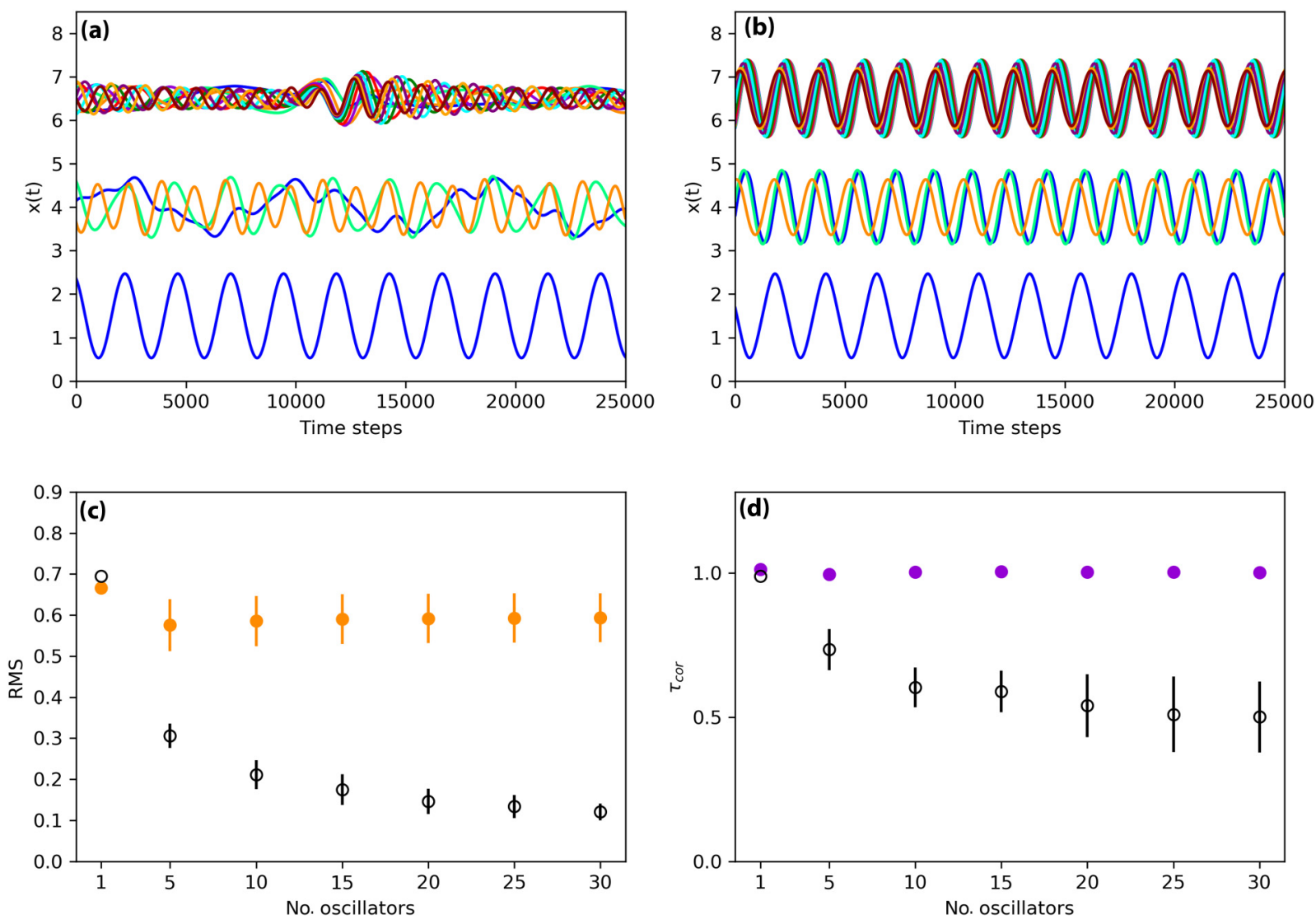

FIG. 2. (a),(b) Time traces of coupled oscillators for the isochronous and nonisochronous $\left(\beta_{\max }=6\right)$ systems, respectively. The bottom, middle, and top sets of traces correspond to system sizes of $N=1,3$, and 10, respectively. (c) Root-mean-square (RMS) amplitude of the autonomous oscillations for a range of system sizes for the isochronous (black open circles) and nonisochronous (orange filled circles) cases. (d) Normalized correlation time [Eq. (6)] for the isochronous (black open circles) and nonisochronous (purple filled circles) systems.

plateau value. We use this method to characterize the response time or temporal resolution of the system. We scale the response time of the nonisochronous system to that of the isochronous system and show that nonisochronicity not only does not degrade the temporal resolution, but in fact slightly enhances the rapidity of the response. Further, the speed of the system is independent of the system size [Figs. 3(c) and 3(f)].

\section{EXPERIMENTAL RESULTS}

To experimentally test our theoretical predictions, we created hybrid systems, in which groups of biological hair cells were artificially coupled by mica flakes of various sizes (see Sec. III). The mica membranes were introduced into the solutions bathing the top surface of the biological epithelia, and allowed to adhere to the underlying hair bundles, thus providing coupling. As the thin sheets of mica are transparent, they allow for precise imaging of the motion of the underlying hair bundles [Figs. 4(a)-4(c)]. Hair bundles often exhibited synchronization, despite dispersion in their natural frequencies as large as fivefold [Figs. 4(d)-4(g)] [28], consistent with our theoretical predictions for nonisochronous oscillators. After recording the motion of the coupled hair bundles, the artificial membranes were removed through fluid exchange. This process did not result in any measurable damage of the prepa- ration and the hair bundles continued to display robust spontaneous oscillations in all experiments [Figs. 5(a) and 5(b)].

We compare the amplitude (root mean square) of the hair bundles' spontaneous oscillations across different sizes of artificial membranes, and hence different sizes of coupled networks [Figs. 5(a) and 5(c)]. Due to the variation in heights of neighboring hair bundles, not every bundle under the membrane makes contact with it or becomes coupled. Therefore, we define a network by considering only those hair bundles that have motion correlated to another bundle in the network. We use a cross-correlation [Eq. (4)] threshold of 0.1 to ensure that every bundle in the network is coupled. As an additional test, we repeat the calculation for a higher cross-correlation threshold of 0.5 , ensuring that all of the oscillators in the network are fully synchronized [Fig. 7(a)]. Figures 4 and 5 show representative traces of fully synchronized hair bundles. For both choices of cross-correlation threshold, we consistently observe that the amplitude of the coupled motion is not reduced with increasing number of hair bundles. This finding is consistent with the behavior of nonisochronous oscillators and contrasts that of the isochronous system. In the isochronous case, coupling 10 oscillators together results in an amplitude reduction of more than threefold as compared to the individual, uncoupled oscillators [Fig. 2(c)]. However, in the nonisochronous case, the amplitude remains constant across all system sizes. 

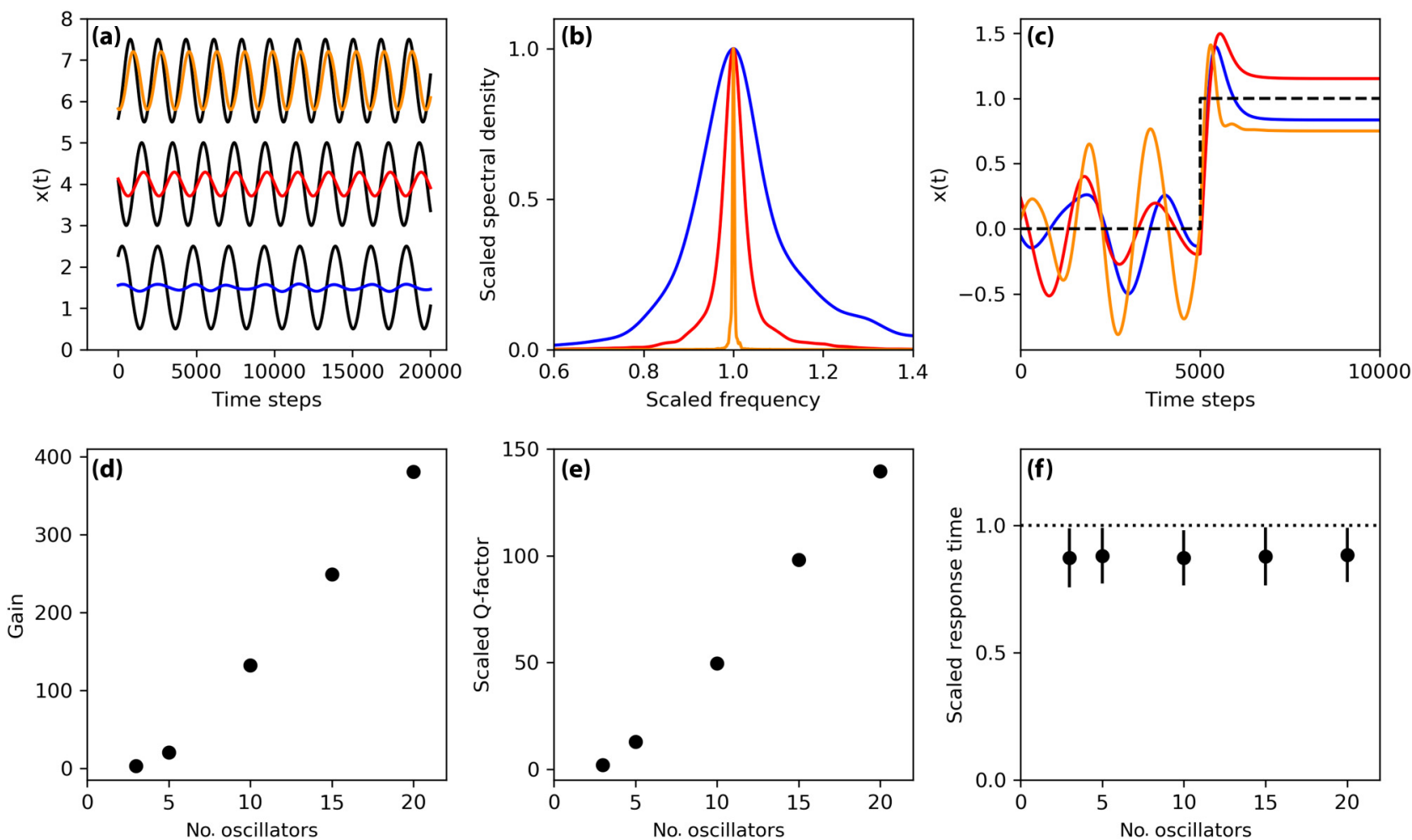

FIG. 3. (a) Average response of the system to weak sinusoidal stimulus, illustrated by the black curves. The stimulus frequency was set to the median limit-cycle frequency of the network, $F(t)=0.05 \sin \left(\Omega_{\frac{N+1}{2}} t\right)$. The responses (blue, red, and orange curves) represent the average over all oscillators, over 20 presentations of the stimulus, each with random initial conditions. Bottom, middle, and top traces correspond to $\beta_{\max }=0,2$, and 6, respectively. (b) Spectral curves in response to low-level white-noise stimulus. The curves get increasingly narrow for increasing $\beta_{\max }$. (c) Average oscillator response to a step stimulus, as indicated by the black dashed curve. Middle, top, and bottom plateau curves correspond to $\beta_{\max }=0,2$, and 6, respectively. For (a)-(c), the system size was $N=10$, and the blue, red, and orange curves represent $\beta_{\max }=0,2$, and 6, respectively. (d) Spectral value of the nonisochronous $\left(\beta_{\max }=6\right)$ system at the resonance frequency in response to weak white-noise stimulus, scaled to the corresponding spectral response of the isochronous system. (e) Quality factor of the system with $\beta_{\text {max }}=6$ in response to weak white-noise stimulus, scaled to the quality factor of the isochronous system. (f) Response time of the nonisochronous $\left(\beta_{\max }=6\right)$ system to a step stimulus, scaled to the response time of the isochronous system. Points and error bars represent the mean and the standard deviation over 100 presentations of the stimulus, each with random initial conditions.

We also compare the coherence [Eq. (6)] of hair bundle oscillations across network sizes of all coupled oscillators [Fig. 5(d)] and of just those displaying synchronization [Fig. 7(b)]. Consistent with the theoretical predictions for coupled nonisochronous oscillators, the coherence does not fall off upon increasing the number of oscillators in the network. In contrast, the coherence of the isochronous system reduces by nearly twofold for a system of 10 oscillators as compared to the individual, uncoupled oscillators [Fig. 2(d)].

\section{DISCUSSION}

Auditory and vestibular systems have provided an experimental testing ground for concepts in nonequilibrium thermodynamics [29], condensed-matter theory [30], and nonlinear dynamics [13]. How active hair cells exhibit notable performance as signal detectors, displaying sensitivity of response, frequency selectivity, and high temporal resolution, all within a noisy fluid environment, is a longstanding open question in this area of study. Further, auditory organs tend to contain overlying structures that impose a strong degree of coupling between individual hair cells, which in turn exhibit dispersion of the characteristic frequencies. It has not been established which role the presence of both strong coupling and significant frequency dispersion play in achieving the detection characteristics, or how the system avoids amplitude death to form clusters of synchronized oscillators necessary for generating SOAEs.

Simulations of our numerical model of coupled hair bundles indicate that the nonisochronicity of the oscillators, which results in chaotic dynamics, is responsible for this robust synchronization. The synchronization yields great enhancement of the system's sensitivity and frequency selectivity to weak external signals. Unlike proximity to the Hopf bifurcation, this enhancement does not come at the cost of reduced temporal resolution. Further, this synchronization persists for large numbers of oscillators and despite large frequency dispersion. Neither the amplitude nor the coherence of the oscillations is reduced upon increasing the number of oscillators. These results are consistent with the remarkable signal-detection attributes of the auditory system and the experimental observations of sharp spectral peaks in the SOAE recordings. 
(a)

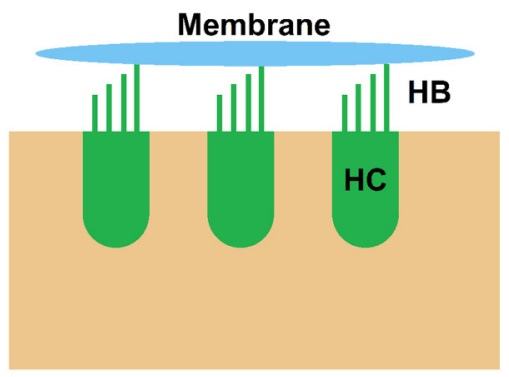

(b)

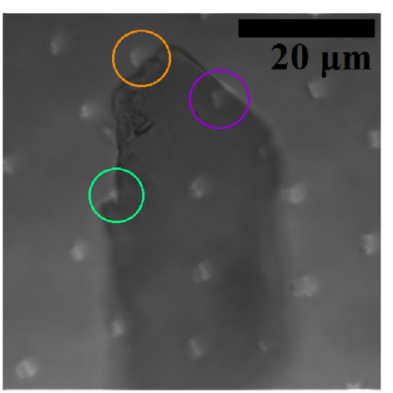

(c)
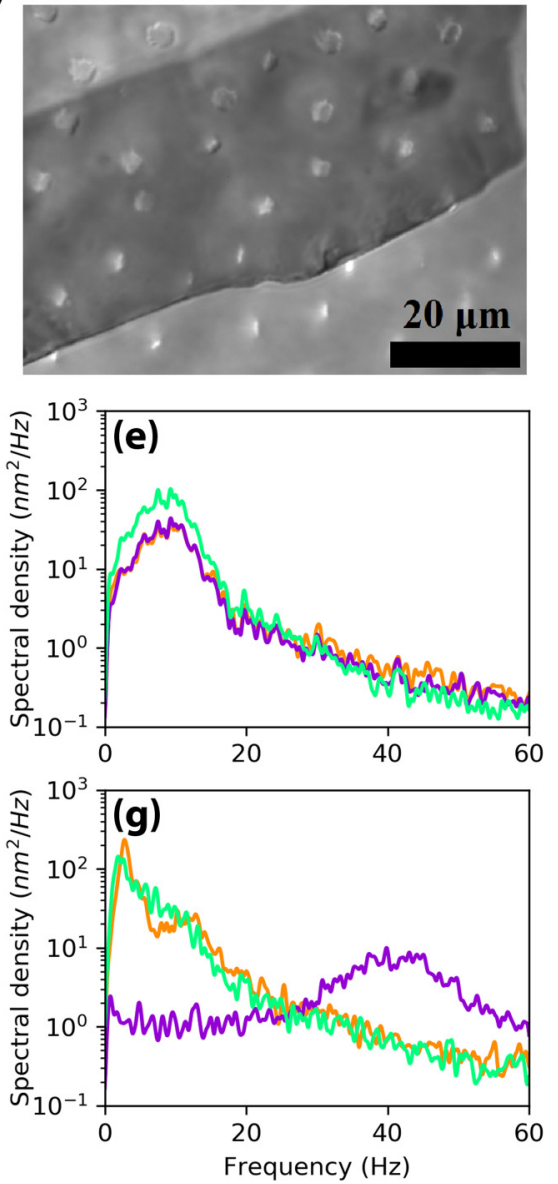

FIG. 4. (a) Illustration of the experimental system from a side view, displaying the hair cells (HC), hair bundles (HB), and an artificial membrane. (b),(c) Top-down images of biological preparations. The hair bundles appear as white dots, and the shadow cast by the transparent artificial membrane can be seen in the center of the images. (d),(e) Time traces and power spectra of three spontaneously oscillating hair bundles coupled by an artificial membrane. These bundles are circled in (b) with colors corresponding to their time traces. The top, middle, and bottom traces correspond to the topmost, leftmost, and bottommost circles in (b). (f),(g) Time traces and power spectra of the three hair bundles in (d) and (e) after removal of the artificial membrane.

The results from our experimental recordings of coupled hair bundles are consistent with those of the numerical model. By coupling various numbers of hair cells with artificial membranes, we find that hair bundles with differences in characteristic frequencies as large as fivefold still routinely synchronize. Further, the amplitude and coherence of the spontaneous oscillations are both independent of the number of hair bundles in the network. These results can be reproduced by the numerical model only when the oscillators are chaotic $\left(\beta_{j} \neq\right.$ $0)$. This suggests that the instabilities that give rise to chaotic dynamics of the individual hair bundles enhance the synchronization and the signal detection of the coupled system.

The coupling strength of the networks of hair bundles in our experiments is likely strong, as it results in synchronization between bundles with vastly different characteristic frequencies. However, frequency dispersion, coupling strength, and topology of the coupling vary greatly across species and sensory organs [6]. The mammalian cochlea, for example, has a tonotopic organization of the hair cells and has a compliant overlying connecting structure, leading to smaller domains of coupling [31]. Therefore, cochlear hair cells would likely not synchronize over a frequency range as large as that observed in our experiments. However, our numerical model suggests that the benefits of synchronization grow rapidly with the number of oscillators [Figs. 3(d) and 3(e)]. Hence, even if synchronization is limited to small groups of hair bundles and over narrow ranges of characteristic frequencies, we expect the improvement in signal detection to be substantial.

Stochastic noise often limits signal detection. However, it has been shown that noise can also enhance the ability of sensory systems, including hair cells, to detect weak signals, through a phenomenon called stochastic resonance [32]. Similarly, low-dimensional chaos is often considered a harmful element in dynamical systems and something to be avoided. For example, a chaotic heartbeat is an indicator of cardiac fibrillation [33]. However, it has also been established that chaotic oscillators can easily synchronize with each other or entrain to an external signal [34,35], as instabilities that give rise to chaotic dynamics can make the oscillators more adaptable to modifications in their autonomous motion. Since biological systems tend to have many degrees of freedom and 

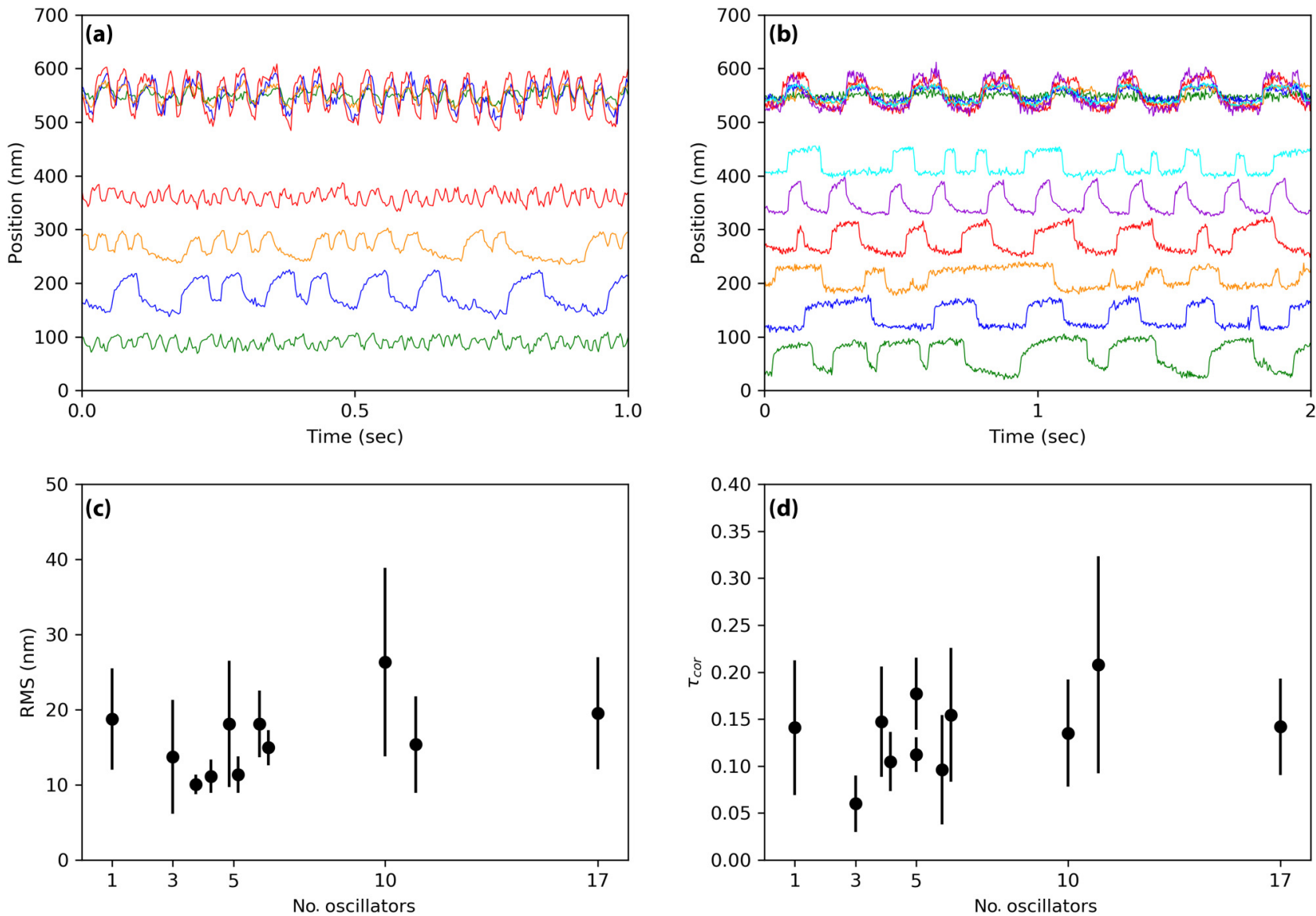

FIG. 5. (a),(b) Overlaid traces of coupled hair bundles (top) for system sizes of $N=4$ and $N=6$, respectively. Below the overlaid traces are the traces of the individual hair bundles obtained in the absence of coupling. (c),(d) RMS amplitude and the normalized correlation time of spontaneous oscillations of coupled hair bundles, obtained for various system sizes. Each hair bundle had a cross-correlation coefficient of at least 0.1 with other bundles in the network. For both panels, points and error bars represent, respectively, the mean and the standard deviation of the coupled oscillators in the system. For systems with $N>1$, each data point represents a separate group of coupled hair bundles. The points and error bars at $N=1$ represent the collective mean and standard deviation across all 10 groups of hair bundles in the absence of coupling. These 10 groups of hair bundles were obtained from four sacculi from four different frogs.

contain nonlinearities, chaos may be a ubiquitous element in their dynamics. We speculate that chaos may be important in other biological systems where timing, sensitivity, and synchronization are desired, especially sensory systems responsible for the detection of external signals.

\section{ACKNOWLEDGMENTS}

The authors gratefully acknowledge the support of NSF Biomechanics and Mechanobiology, under Grant No. 1916136. The authors thank Dr. Sebastiaan Meenderink for developing the software used for tracking hair bundle movement.

\section{APPENDIX}

\section{Approximation of artificial membrane drag}

We approximate the artificial membranes as infinitely thin circular disks. Due to the low Reynolds number of hair bundle dynamics, we assume the system obeys Stokes' law. The
Stokes' drag of an infinitely thin circular disk moving edgewise through an infinite fluid is given by

$$
\lambda_{s}=\frac{16 \eta d}{3},
$$

where $\eta$ is the dynamic viscosity of the fluid and $d$ is the diameter of the disk [36]. We use the viscosity of water, $\eta \approx 10^{-3} \mathrm{~Pa} \mathrm{~s}$, and our approximate experimental range of membrane diameters $(20-50 \mu \mathrm{m})$. The diameters of the membranes are significantly larger than their thicknesses $(<$ $1 \mu \mathrm{m})$, so we consider the infinitely thin disk to be a reasonable approximation. Further, the boundary of the fluid is $\approx 1 \mathrm{~cm}$ away from the structures of interest, which is much farther than the length scale of the membranes, so the assumption of an infinite fluid is reasonable.

We, therefore, approximate the drag coefficients of the artificial membranes to be $\lambda_{s} \approx 100-250 \mathrm{nN} \mathrm{s} \mathrm{m}^{-1}$. We compare this value to the drag coefficient of individual freestanding hair bundles. It has previously been shown that most of the drag contribution of hair bundles comes from the 


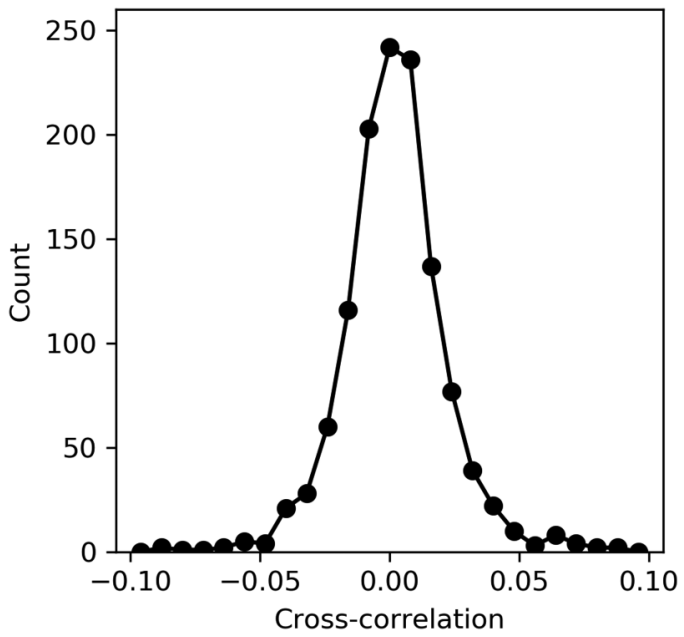

FIG. 6. Histogram of the cross-correlation coefficients between pairs of uncoupled, spontaneously oscillating hair bundles (1225 unique pairs). The standard deviation of this distribution is $<0.02$ and no points exceed 0.1 .

channel-gating friction [37]. The lower bound on the total drag coefficient of an individual freestanding hair bundle was estimated to be $\lambda_{0}=425 \pm 70 \mathrm{nN} \mathrm{s} \mathrm{m}^{-1}$. Therefore, in our numerical simulations, we use a small value for the membrane drag $(\lambda=0.1)$, as it contributes only minimally to the drag of the entire coupled system.

\section{Cross-correlation coefficient noise floor}

Figure 6 provides a histogram of the cross-correlation coefficients between pairs of uncoupled, spontaneously oscillating hair bundles.

\section{Amplitude and coherence of synchronized hair bundles}

Figure 7 provides the RMS amplitude and normalized correlation time of spontaneous oscillations of coupled hair bundles for various system sizes.
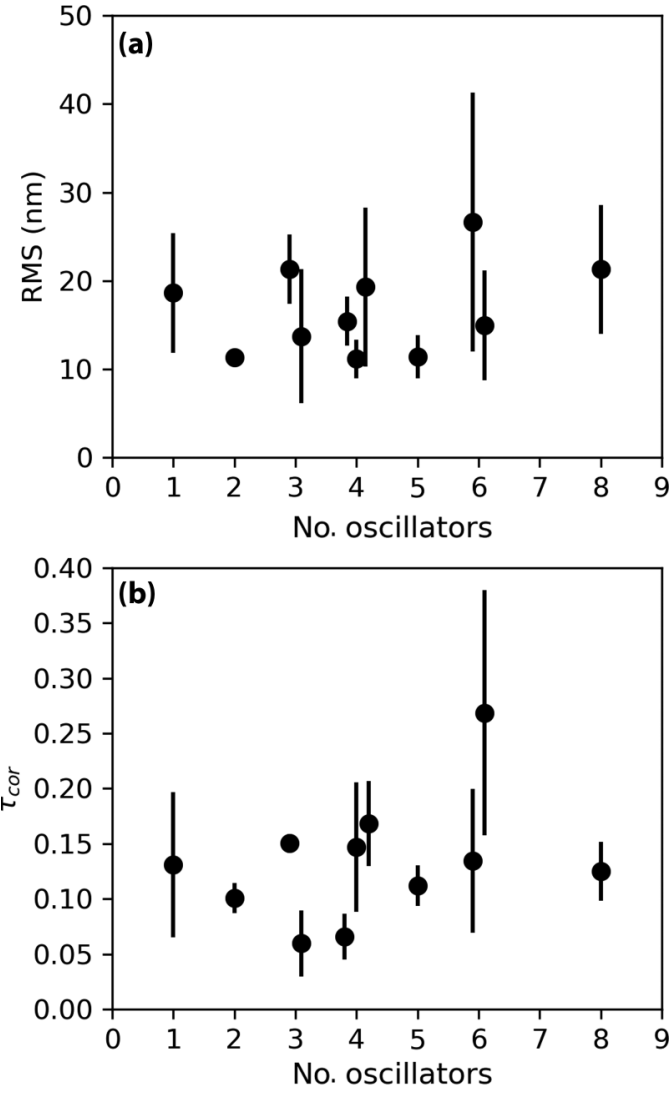

FIG. 7. (a) RMS amplitude and (b) normalized correlation time of spontaneous oscillations of coupled hair bundles, obtained for various system sizes. Each hair bundle had a cross-correlation coefficient of at least 0.5 with other bundles in the network in order to ensure the network is synchronized. For both panels, points and error bars represent, respectively, the mean and the standard deviation of the coupled oscillators in the system. For systems with $N>1$, each data point represents a separate group of coupled hair bundles. The points and error bars at $N=1$ represent the collective mean and standard deviation across all 10 groups of hair bundles in the absence of coupling.
[1] A. J. Hudspeth, Integrating the active process of hair cells with cochlear function, Nat. Rev. Neurosci. 15, 600 (2014).

[2] B. Leshowitz, Measurement of the twoclick threshold, J. Acoust. Soc. Am. 49, 462 (1971).

[3] T. Reichenbach and A. J. Hudspeth, The physics of hearing: Fluid mechanics and the active process of the inner ear, Rep. Prog. Phys. 77, 076601 (2014).

[4] M. LeMasurier and P. G. Gillespie, Hair-cell mechanotransduction and cochlear amplification, Neuron 48, 403 (2005).

[5] M. A. Vollrath, K. Y. Kwan, and D. P. Corey, The micromachinery of mechanotransduction in hair cells, Annu. Rev. Neurosci. 30, 339 (2007).

[6] D. Ó Maoiléidigh and A. J. Ricci, A bundle of mechanisms: Inner-ear hair-cell mechanotransduction, Trends Neurosci. 42, 221 (2019).
[7] A. J. Hudspeth, Making an effort to listen: Mechanical amplification in the ear, Neuron 59, 530 (2008).

[8] M. E. Benser, R. E. Marquis, and A. J. Hudspeth, Rapid, active hair bundle movements in hair cells from the bullfrogs sacculus, J. Neurosci. 16, 5629 (1996).

[9] P. Martin, D. Bozovic, Y. Choe, and A. J. Hudspeth, Spontaneous oscillation by hair bundles of the bullfrog's sacculus, J. Neurosci. 23, 4533 (2003).

[10] A. C. Crawford and R. Fettiplace, The mechanical properties of ciliary bundles of turtle cochlear hair cells, J. Physiol. 364, 359 (1985).

[11] P. Martin, A. J. Hudspeth, and F. Jülicher, Comparison of a hair bundle's spontaneous oscillations with its response to mechanical stimulation reveals the underlying active process, Proc. Natl. Acad. Sci. USA 98, 14380 (2001). 
[12] P. Martin and A. J. Hudspeth, Active hair-bundle movements can amplify a hair cell's response to oscillatory mechanical stimuli, Proc. Natl. Acad. Sci. USA 96, 14306 (1999).

[13] V. M. Eguiluz, M. Ospeck, Y. Choe, A. J. Hudspeth, and M. O. Magnasco, Essential Nonlinearities in Hearing, Phys. Rev. Lett. 84, 5232 (2000).

[14] A. Kern and R. Stoop, Essential Role of Couplings Between Hearing Nonlinearities, Phys. Rev. Lett. 91, 128101 (2003).

[15] S. Ji, D. Bozovic, and R. Bruinsma, Amphibian sacculus and the forced Kuramoto model with intrinsic noise and frequency dispersion, Phys. Rev. E 97, 042411 (2018).

[16] J. Faber and D. Bozovic, Chaotic dynamics enhance the sensitivity of inner ear hair cells, Sci. Rep. 9, 18394 (2019).

[17] J. Faber and D. Bozovic, Chaotic dynamics of inner ear hair cells, Sci. Rep. 8, 3366 (2018).

[18] D. T. Kemp, Evidence of mechanical nonlinearity and frequency selective wave amplification in the cochlea, Arch. Oto-Rhino-Laryngol. 224, 37 (1979).

[19] Y. Roongthumskul, D. Ó Maoiléidigh, and A. J. Hudspeth, Bilateral spontaneous otoacoustic emissions show coupling between active oscillators in the two ears, Biophys. J. 116, 2023 (2019).

[20] A. Vilfan and T. Duke, Frequency clustering in spontaneous otoacoustic emissions from a lizard's ear, Biophys. J. 95, 4622 (2008).

[21] F. Fruth, F. Jülicher, and B. Lindner, An active oscillator model describes the statistics of spontaneous otoacoustic emissions, Biophys. J. 107, 815 (2014).

[22] K.-J. Kim and K.-H. Ahn, Amplitude death of coupled hair bundles with stochastic channel noise, Phys. Rev. E 89, 042703 (2014).

[23] J. Faber and D. Bozovic, Noise-induced chaos and signal detection by the nonisochronous Hopf oscillator, Chaos 29, 043132 (2019).

[24] Y. Roongthumskul, J. Faber, and D. Bozovic, Dynamics of mechanically coupled hair-cell bundles of the inner ear, Biophys. J. 120, 205 (2021).

[25] N. Ciganović, A. Wolde-Kidan, and T. Reichenbach, Hair bundles of cochlear outer hair cells are shaped to minimize their fluid-dynamic resistance, Sci. Rep. 7, 3609 (2017).
[26] A. S. Pikovsky and J. Kurths, Coherence Resonance in a Noise-Driven Excitable System, Phys. Rev. Lett. 78, 775 (1997).

[27] K. Dierkes, F. Jülicher, and B. Lindner, A mean-field approach to elastically coupled hair bundles, Eur. Phys. J. E 35, 37 (2012).

[28] T.-Y. Zhang, S. Ji, and D. Bozovic, Synchronization of spontaneous active motility of hair cell bundles, PLoS One $\mathbf{1 0}$ e0141764 (2015).

[29] L. Dinis, P. Martin, J. Barral, J. Prost, and J. F. Joanny, Fluctuation-Response Theorem for the Active Noisy Oscillator of the Hair-Cell Bundle, Phys. Rev. Lett. 109, 160602 (2012).

[30] T. Risler, J. Prost, and F. Jülicher, Universal Critical Behavior of Noisy Coupled Oscillators, Phys. Rev. Lett. 93, 175702 (2004).

[31] D. M. Freeman, C. C. Abnet, W. Hemmert, B. S. Tsai, and T. F. Weiss, Dynamic material properties of the tectorial membrane: A summary, Hear. Res. 180, 1 (2003).

[32] F. Jaramillo and K. Wiesenfeld, Mechanoelectrical transduction assisted by Brownian motion: A role for noise in the auditory system, Nat. Neurosci. 1, 384 (1998).

[33] A. Garfinkel, P.-S. Chen, D. O. Walter, H. S. Karagueuzian, B. Kogan, S. J. Evans, M. Karpoukhin, C. Hwang, T. Uchida, M. Gotoh, O. Nwasokwa, P. Sager, and J. N. Weiss, Quasiperiodicity and chaos in cardiac fibrillation, J. Clin. Invest. 99, 305 (1997).

[34] A. Garfinkel, M. L. Spano, W. L. Ditto, and J. N. Weiss, Controlling cardiac chaos, Science 257, 1230 (1992).

[35] A. B. Neiman, K. Dierkes, B. Lindner, L. Han, and A. L. Shilnikov, Spontaneous voltage oscillations and response dynamics of a Hodgkin-Huxley type model of sensory hair cells, J. Math. Neurosci. 1, 11 (2011).

[36] J. F. Trahan, Stokes drag on a thin circular disk moving edgewise midway between parallel plane boundaries, J. Fluids Eng. 128, 887 (2006).

[37] V. Bormuth, J. Barral, J.-F. Joanny, F. Jülicher, and P. Martin, Transduction channels' gating can control friction on vibrating hair-cell bundles in the ear, Proc. Natl. Acad. Sci. USA 111, 7185 (2014). 